\title{
Elevated Neutrophil-to-Lymphocyte Ratio in Perioperative Periods is Suggestive of Poor Prognosis in Patients with Colorectal Cancer
}

\author{
Jung Hyun Kim ' \\ Jae-Hoon Lee ${ }^{2}$ \\ Hye Sun Lee $\mathbb{D}^{3}$ \\ Su-Jin Shin ${ }^{4}$ \\ Eun Jung Park' \\ Eun-Suk Cho ${ }^{5}$ \\ Seung Hyuk Baik' \\ Kang Young Lee ${ }^{6}$ \\ Jeonghyun Kang (1D' \\ 'Department of Surgery, Gangnam \\ Severance Hospital, Yonsei University \\ College of Medicine, Seoul, Republic of \\ Korea; ${ }^{2}$ Department of Nuclear \\ Medicine, Gangnam Severance Hospital, \\ Yonsei University College of Medicine, \\ Seoul, Republic of Korea; ${ }^{3}$ Biostatistics \\ Collaboration Unit, Yonsei University \\ College of Medicine, Seoul, Republic of \\ Korea; ${ }^{4}$ Department of Pathology, \\ Gangnam Severance Hospital, Yonsei \\ University College of Medicine, Seoul, \\ Republic of Korea; ${ }^{5}$ Department of \\ Radiology, Gangnam Severance Hospital, \\ Yonsei University College of Medicine, \\ Seoul, Republic of Korea; ${ }^{6}$ Department of \\ Surgery, Severance Hospital, Yonsei \\ University College of Medicine, Seoul, \\ Republic of Korea
}

Correspondence: Jeonghyun Kang Department of Surgery, Gangnam Severance Hospital, Yonsei University College of Medicine, 2II Eonju-ro, Gangnam-gu, Seoul, 06273, Republic of Korea

Tel +82-2-2019-3372

Fax +82-2-3462-5994

Email ravic@naver.com
Background: Recent data suggest that alterations in the neutrophil-to-lymphocyte ratio (NLR) in the perioperative periods can serve as prognostic factors. However, research on the clinical impact has been limited and even discordant in patients with colorectal cancer (CRC).

Patients and Methods: The optimal cut-off value of preoperative NLR (NLR-pre), postoperative NLR (NLR-post), and its change (NLR-delta) were determined to maximize differences in overall survival (OS) between groups. Patients were categorized into four groups (NLR-trend) as follows: G1, low NLR-pre and NLR-post; G2, low NLR-pre and high NLR-post; G3, high NLR-pre and low NLR-post; and G4, high NLR-pre and NLR-post. Discriminatory performance was compared using integrated AUC (iAUC) between all indicators.

Results: A total of 576 patients diagnosed with stage I-IV CRC were included. The cut-off points were determined as 2.33 for NLR-pre, 2.06 for NLR-post, and -1.08 for NLR-delta. Subgroup dichotomization using NLR-pre, NLR-post, NLR-delta and NLR-trend were all identified as significant prognostic factors by univariate analysis. However, NLR-trend was only remained as an independent prognostic factor in the multivariate analysis. The iAUC of the NLR-trend was superior to that of NLR-pre (bootstrap iAUC mean difference $=0.036$; 95\% CI 0.013-0.073), NLR-post (bootstrap iAUC mean difference $=0.045$; 95\% CI 0.019 0.081 ) and NLR-delta (bootstrap iAUC mean difference $=0.061 ; 95 \%$ CI $0.025-0.104$ ).

Conclusion: Risk stratification and combining of preoperative and postoperative NLR (NLR-trend) can improve prognostic discrimination compared with single measurements or simple changes in NLR in patients with CRC.

Keywords: NLR, colorectal cancer, survival, iAUC

\section{Introduction}

Colorectal cancer (CRC) is one of the most common gastrointestinal malignancies, both world and in Korea. ${ }^{1,2}$ It is very important to predict the prognosis of patients prognosis in order to best implement adjuvant chemotherapy or radiotherapy. Although clinical, pathologic, and molecular parameters that can predict prognosis have been evaluated, ${ }^{3,4}$ convenient and inexpensive predictors of clinical outcome in CRC nature have not yet been clearly introduced.

Systemic inflammation has been shown to be associated with long-term survival outcomes in various type of cancer patients. ${ }^{5,6}$ The neutrophil-tolymphocyte ratio (NLR) is one of the most measured and evaluated indicators 
due to the advantage of being easy to obtain through routine blood test, with a relatively low cost. Previous studies investigating the usefulness of NLR analyzed its clinical impact using either a preoperative (NLR-pre) or postoperative NLR (NLR-post). ${ }^{7,8}$ Recently, it was argued that the combination of preoperative and postoperative systemic inflammatory markers could be independently associated with overall survival (OS) or recurrence-free survival. ${ }^{9,10}$ Similarly, some investigators have tried to consider sequential changes in NLR (NLR-post minus NLR-pre), which is known as the NLR-delta. NLR-delta has been demonstrated as an alternative prognostic factor in patients with non-small cell lung cancer, hepatocellular carcinoma, gastric cancer, and anaplastic thyroid cancer. ${ }^{11-16}$

However, there are still some discrepancies in the clinical significance of grouping the NLR-pre and NLP-post ${ }^{9,10}$ and the clinical significance of NLR-delta in patients with CRC also showed conflicting results. ${ }^{17,18}$ In addition, the significance of combining pre- and post-operative NLR (NLR-trend) and NLR-delta has rarely been evaluated simultaneously, and it remains unclear whether there is a difference in the strength of association with survival between the two dynamic matrices.

When previous studies analyzed longitudinal NLR changes, preoperative NLR was usually measured within 1 month prior to surgery. However, re-measuring periods of postoperative NLR differ from study to study, which makes it difficult to generalize the results. A recent study showed that the inflammatory indices measured between 3 and 8 weeks after surgery are values measured in a state that minimizes inflammation related to surgery. ${ }^{10}$ This period also coincides with the recommended initiation time for receipt of chemotherapy after surgical resection in patients with CRC. ${ }^{19,20}$ Therefore, the NLR value measured over 38 week periods could have a clinical advantage in deciding the implementation of postoperative chemotherapy.

The aim of the present study was to investigate and compare the clinical impact of trajectory change of NLR from preoperative NLR and postoperative NLR measured during 3 to 8 weeks postoperative periods in patients with CRC.

\section{Patients and Methods}

\section{Patients}

This was a retrospective, single-institution study that included patients diagnosed with stage I to IV CRC who underwent surgery with curative-intent between
January 2004 and April 2014 at Gangnam Severance Hospital, Yonsei University College of Medicine.

The inclusion criteria were as follows: (1) patients who underwent surgical resection of stage I-IV CRC, (2) no previous chemotherapy or radiation treatment before surgery, and (3) available neutrophil and lymphocyte counts both in the preoperative period within 31 days before surgery and postoperative periods between 21 and 56 days after surgery. The exclusion criteria were as follows: (1) insufficient data of stage, (2) histology other than adenocarcinoma such as neuroendocrine tumor, gastrointestinal stromal tumors, and other types of cancers, (3) appendiceal cancer or anal cancer, (4) hereditary nonpolyposis colorectal cancer or familial adenomatous polyposis associated cancers, (5) inflammatory bowel disease, (6) postoperative blood sample acquired after initiation of postoperative chemotherapy, and (7) postoperative blood sample acquired before discharge date due to delayed hospitalization. According to our inclusion and exclusion criteria, 567 patients were included in this study (Supplementary Figure S1).

All procedures performed in studies involving human participants were in accordance with the ethical standards of the institutional and/or national research committee and with the 1964 Declaration of Helsinki and its later amendments or comparable ethical standards. The institutional ethics committee of Gangnam Severance Hospital, Yonsei University College of Medicine approved this study, and the need for informed consent was waived because of its retrospective design.

\section{Definition of NLR-Pre, NLR-Post, NLR-Delta, and NLR-Trend}

Neutrophil and lymphocyte counts were measured using routine laboratory systems. NLR was calculated as the absolute neutrophil count divided by the absolute lymphocyte count. Blood samples were obtained preoperatively within 31 days prior to surgical resection and postoperatively 21-56 days after surgery. NLR values measured at these time points were defined as NLR-pre and NLR-post, respectively. NLRdelta was defined as NLR-post minus NLR-pre. In addition, patients were allocated into the high or low NLR groups based on the cut-off values of NLR-pre and NLR-post, respectively. Using the subgroups defined in this way, patients were divided into four groups (NLR-trend) as follows: G1, low preoperative and low postoperative NLR; G2, low preoperative but high postoperative NLR; G3, high 
preoperative but low postoperative NLR; G4, high preoperative and high postoperative NLR.

\section{Follow-Up of Patients}

All patients were followed regularly every 3-6 months for the first 3 years and every 6 months thereafter. Follow-up examinations routinely included physical examination and blood laboratory tests, including carcinoembryonic antigen (CEA) tests. In most patients, abdominopelvic computed tomography (CT) and/or chest CT were performed every 6 months. Other examinations, such as colonoscopy, pelvic magnetic resonance imaging, or ${ }^{18} \mathrm{~F}$-fluorodeoxyglucose positron-emission tomography, were performed at the physician's discretion. Patients were followed until October 2019 or death of the patient. The median follow-up period was 89 months (interquartile range [IQR], 61-110 months).

\section{Statistical Analysis}

OS was defined as the time from the date of surgery to the date of death from any cause or date of the last follow-up. To analyze the hazard ratio (HR) and $95 \%$ confidence interval (CI) for factors associated with OS, a Cox proportional hazard model was used. Among factors related to OS, only variables found to be significant $(p<0.01)$ in the univariate Cox regression analysis were included in the multivariate analysis.

The X-tile program was used to set the optimal cut-off values of NLR-pre, NLR-post, and NLR-delta for predicting $\mathrm{OS}^{21}{ }^{21}$ Kaplan-Meier curves and Log rank tests were used to compare survival outcomes between the groups.

The predictive accuracy of the model during the follow-up period was measured using the integrated area under the curve (iAUC), which is a weighted mean of AUC over the follow-up period. Differences in accuracy between NLR-derived predictive models were calculated using a bootstrapping method with resampling performed 1000 times. A higher iAUC indicates a higher predictive accuracy for the model.

Statistical analysis was performed using $\mathrm{R}$ version 3.6.3 (R-project, Institute for Statistics and Mathematics, Vienna, Austria). Statistical significance was set at $p<0.05$.

\section{Results}

A total of 567 patients were included in the study. The median age was 63 (IQR, 55-70) years; 342 (60.3\%) were men and $225(39.7 \%)$ were women. During the study period, 177 patients $(31.2 \%)$ died. The median values of NLR-pre and NLR-post were 2.27 (IQR, 1.653-3.124) and 1.832 (IQR, 1.347-2.472), respectively. The median value of NLR-delta was -0.427 (IQR, -1.164-0.228).

\section{Optimal Cut-Off Values of NLR Related}

\section{Parameters}

The cut-off values of NLR-pre and NLR-post that showed the largest $\chi^{2}$ in the Mantel-Cox test were set as 2.33, and 2.06, respectively. Patients were divided into low and high NLR groups according to the cut-off values. Among the 567 patients, 273 (48.1\%) and 219 (38.6\%) patients were allocated to the high NLR group in the preoperative and postoperative periods, respectively. The optimal cut-off value of NLR-delta was also calculated in the same way, and was defined as -1.08 (Supplementary Figure S2).

\section{Comparison of Patient Characteristics According to the Low and High NLRs}

There were significant differences in sex and, preoperative CEA between the low NLR-pre and the high NLR-pre groups. No significant differences were found in age, body mass index (BMI), tumor location, histologic grade, lymphovascular invasion (LVI), complication, stage, or receipt of chemotherapy between the two groups. Conversely, for postoperative NLR, there were significant differences in sex, age, histologic grade, and complications between the low NLRpost and high NLR-post groups (Table 1).

\section{Kaplan-Meier Curves According to NLR-Pre, NLR-Post, NLR-Delta, and NLR-Trend}

There was a significant difference in OS between the low and high NLR-pre groups $(p<0.001)$ (Figure 1A). The 5 -year OS rate was significantly lower in the high NLRpost group than in the low NLR-post group $(p=0.003)$ (Figure 1B). Significant differences were observed with regard to OS among the four groups defined as NLRtrend. Among the four groups, G1, which was defined as persistently low preoperative and postoperative NLRs, was significantly associated with better OS than the other groups (Figure 1C). The low NLR-delta group showed worse OS than the high NLR-delta group (Figure 1D).

\section{Univariate and Multivariate Analysis of OS}

Univariate Cox regression analysis found that sex (HR 1.59, 95\% CI 1.15-2.20, $p=0.004$ ), an age (HR 1.66, 95\% CI 1.232.23, $p=0.0008$ ), CEA (HR 1.86, 95\% CI 1.37-2.52, $p<0.001$ ), LVI (HR $2.09,95 \%$ CI $1.52-2.87, p<0.001$ ), stage IV (versus stage I\&II, HR 5.23, 95\% CI 3.54-7.73, 
Table I Characteristics of Patients $(n=567)$

\begin{tabular}{|c|c|c|c|c|c|c|c|}
\hline & & \multicolumn{2}{|c|}{ NLR-Pre } & \multirow[t]{2}{*}{$p$} & \multicolumn{2}{|c|}{ NLR-Post } & \multirow[t]{2}{*}{$p$} \\
\hline & & Low $(n=294) N(\%)$ & High (n=273) N (\%) & & Low $(n=348) N(\%)$ & High (n=219) N (\%) & \\
\hline Sex & $\begin{array}{l}\text { Female } \\
\text { Male }\end{array}$ & $\begin{array}{l}134(45.6) \\
160(54.4)\end{array}$ & $\begin{array}{l}91(33.3) \\
182(66.7)\end{array}$ & 0.004 & $\begin{array}{l}150(43.1) \\
198(56.9)\end{array}$ & $\begin{array}{c}75(34.2) \\
144(65.8)\end{array}$ & 0.044 \\
\hline Age (years) & $\begin{array}{l}<65 \\
\geq 65\end{array}$ & $\begin{array}{l}170(57.8) \\
124(42.2)\end{array}$ & $\begin{array}{l}139(50.9) \\
134(49.1)\end{array}$ & 0.117 & $\begin{array}{l}212(60.9) \\
136(39.1)\end{array}$ & $\begin{array}{c}97(44.3) \\
122(55.7)\end{array}$ & $<0.001$ \\
\hline BMI $\left(\mathrm{kg} / \mathrm{m}^{2}\right)$ & $\begin{array}{l}<25 \\
\geq 25 \\
\text { No data }\end{array}$ & $\begin{array}{c}205(69.7) \\
86(29.3) \\
3(1)\end{array}$ & $\begin{array}{c}196(71.8) \\
75(27.5) \\
2(0.7)\end{array}$ & 0.828 & $\begin{array}{c}234(67.2) \\
111(31.9) \\
3(0.9)\end{array}$ & $\begin{array}{c}167(76.3) \\
50(22.8) \\
2(0.9)\end{array}$ & 0.066 \\
\hline CEA $(\mathrm{ng} / \mathrm{mL})$ & $\begin{array}{l}<5 \\
\geq 5 \\
\text { No data }\end{array}$ & $\begin{array}{c}187(63.6) \\
93(31.6) \\
14(4.8)\end{array}$ & $\begin{array}{c}152(55.7) \\
113(41.4) \\
8(2.9)\end{array}$ & 0.04 & $\begin{array}{c}221(63.5) \\
113(32.5) \\
14(4)\end{array}$ & $\begin{array}{c}118(53.9) \\
93(42.5) \\
8(3.7)\end{array}$ & 0.054 \\
\hline Tumor location & $\begin{array}{l}\text { Colon } \\
\text { Rectum }\end{array}$ & $\begin{array}{c}217(73.8) \\
77(26.2)\end{array}$ & $\begin{array}{c}203(74.4) \\
70(25.6)\end{array}$ & 0.958 & $\begin{array}{c}260(74.7) \\
88(25.3)\end{array}$ & $\begin{array}{c}160(73.1) \\
59(26.9)\end{array}$ & 0.753 \\
\hline Histologic grade & $\begin{array}{l}\text { G1 \& G2 } \\
\text { G3 } \\
\text { Mucinous \& SRC }\end{array}$ & $\begin{array}{c}27 \mathrm{I}(92.2) \\
12(4.1) \\
11(3.7)\end{array}$ & $\begin{array}{c}248(90.8) \\
8(2.9) \\
17(6.2)\end{array}$ & 0.312 & $\begin{array}{c}323(92.8) \\
7(2) \\
18(5.2)\end{array}$ & $\begin{array}{c}196(89.5) \\
14(5.9) \\
10(4.6)\end{array}$ & 0.047 \\
\hline LVI & $\begin{array}{l}\text { Absent } \\
\text { Present } \\
\text { No data }\end{array}$ & $\begin{array}{l}191(65) \\
81(27.6) \\
22(7.5)\end{array}$ & $\begin{array}{c}173(63.4) \\
88(32.2) \\
12(4.4)\end{array}$ & 0.187 & $\begin{array}{c}231(66.4) \\
97(27.9) \\
20(5.7)\end{array}$ & $\begin{array}{c}133(60.7) \\
72(32.9) \\
14(6.4)\end{array}$ & 0.389 \\
\hline Complications & $\begin{array}{l}\text { No } \\
\text { Yes }\end{array}$ & $\begin{array}{c}233(79.3) \\
61(20.7)\end{array}$ & $\begin{array}{l}211(77.3) \\
62(22.7)\end{array}$ & 0.642 & $\begin{array}{c}287(82.5) \\
61(17.5)\end{array}$ & $\begin{array}{l}157(71.7) \\
62(28.3)\end{array}$ & 0.003 \\
\hline AJCC Stage & $\begin{array}{l}\text { l } \\
\text { III } \\
45(15.3)\end{array}$ & $\begin{array}{c}\&|| \\
|3|(44.6) \\
50(18.3)\end{array}$ & $\begin{array}{l}118(40.1) \\
130(47.6)\end{array}$ & $\begin{array}{l}93(34.1) \\
53(15.2)\end{array}$ & $\begin{array}{c}0.293 \\
162(46.6) \\
42(19.2)\end{array}$ & $\begin{array}{l}133(38.2) \\
99(45.2)\end{array}$ & $78(35.6)$ \\
\hline Chemotherapy & $\begin{array}{l}\text { No } \\
\text { Yes }\end{array}$ & $\begin{array}{c}93(31.6) \\
201(68.4)\end{array}$ & $\begin{array}{l}72(26.4) \\
201(73.6)\end{array}$ & 0.199 & $\begin{array}{l}101(29) \\
247(71)\end{array}$ & $\begin{array}{c}64(29.2) \\
155(70.8)\end{array}$ & $>0.99$ \\
\hline NLR-delta & Median (IQR) & $-0.01(-0.42,0.62)$ & $-1.19(-1.97,-0.52)$ & $<0.001$ & $-0.65(-1.39,-0.15)$ & $0.14(-0.79,0.95)$ & $<0.001$ \\
\hline
\end{tabular}

Abbreviations: BMI, body mass index; CEA, carcinoembryonic antigen; SRC, signet ring cell; LVI, lymphovascular invasion; AJCC, American Joint Committee on Cancer; $\mathrm{HR}$, hazard ratio; $\mathrm{Cl}$, confidence interval.

$p<0.001$ ), complications (HR 1.43, 95\% CI 1.02-1.99, $p=0.036$ ), chemotherapy (HR $0.39,95 \%$ CI $0.29-0.52$, $p<0.001$ ), NLR-pre (HR 1.67, 95\% CI 1.24-2.26, $p=0.0007$ ), NLR-post (HR 1.56, 95\% CI 1.16-2.1, $p=0.003$ ), NLR-trend and NLR-delta (HR $0.68,95 \%$ CI $0.5-0.94, p=0.02$ ) were significantly associated with OS (Table 2). Subsequent multivariate Cox regression identified LVI present vs absent (HR 1.45, 95\% CI 1.04-2.04, $p=0.027$ ), stage [stage III vs stage I \& II (HR 1.9, 95\% CI $1.23-2.94, p=0.003$, stage IV vs stage I \& II (HR 4.11, 95\% CI $2.67-6.33, p<0.001$ )], chemotherapy (HR $0.37,95 \%$ CI $0.26-0.53, p<0.001$ ), and NLR-trend as independent risk factors for OS (Table 2). The clinical significance of NLRtrend was confirmed in a subgroup analysis performed using stage II and stage III patients. (Supplementary Table 1).

\section{Integrated AUC}

Throughout the observation period, the integrated AUC value of NLR-trend $(0.599,95 \% \mathrm{CI}=0.562-0.637)$ was superior to that of NLR-pre $(0.562,95 \% \mathrm{CI}=0.524-0.599)$ (bootstrap iAUC mean difference $=0.036,95 \% \mathrm{CI}=0.013-0.073$ ), NLRpost $(0.553,95 \% \mathrm{CI}=0.516-0.589)$ (bootstrap iAUC mean difference $=0.045,95 \% \mathrm{CI}=0.019-0.081$ ), and NLR-delta (0.537, 95\% $\mathrm{CI}=0.504-0.570)$ (bootstrap iAUC mean difference $=0.061,95 \% \mathrm{CI}=0.025-0.104$ ), respectively (Figure 2).

\section{Discussion}

This study demonstrated that the combinational stratification using trajectory change of NLR (NLR-trend) has superior prognostic performance than using NLR 

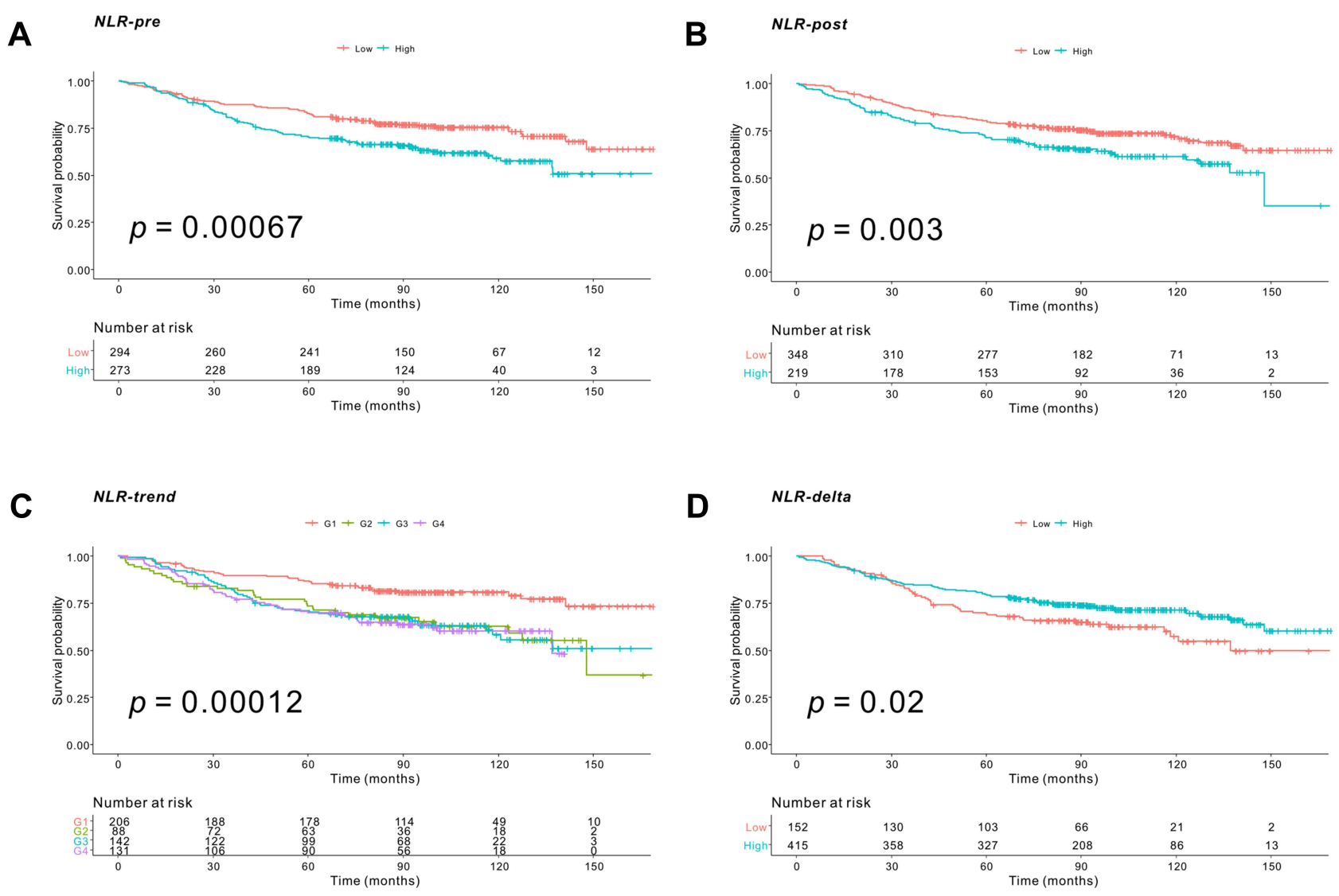

Figure I Kaplan-Meier survival curve of neutrophil-to-lymphocyte ratio related parameters for overall survival of CRC patients. (A) NLR-pre; The low NLR-pre group (NLR-pre $\leq 2.33$ ) had better OS compared to high NLR-pre group (NLR-pre >2.33) (5 year OS: 82.6\% versus $70.5 \%$; $p<0.001$ ). (B) NLR-post; The low NLR-post group (NLR-post $\leq 2.06$ ) was associated with better OS compared to high NLR-post group (NLR-post >2.06) (5 year OS: $80.1 \%$ versus $71.4 \%$; $p=0.003$ ). (C) NLR-trend; The GI (low NLR-pre and low NLR-post group) was significantly associated with better OS than the other groups (low NLR-pre and high NLR-post group, high NLR-pre and low NLR-post group and high NLR-pre) (5 year OS: 86.4\%, 73.6\%, 70.2\% and 70.0\% respectively, $p=0.001$ ). (D) NLR-delta; The low NLR-delta group (NLR-delta $\leq-1.08$ ) had worse OS compared to high NLR-delta group (NLR-delta >-1.08). (5 year OS: $69.5 \%$ versus $79.4 \% ; p=0.003$ ).

measured either in the preoperative or postoperative period, or the NLR-delta value in patients with CRC.

Among the various inflammatory markers, NLR is known as a serum biomarker useful in predicting prognosis. ${ }^{22}$ Neutrophils activate endothelial and parenchymal cells and generate various reactive oxygen species, causing DNA damage, ${ }^{23}$ as well as creating various mediators that promote cancer proliferation and metastasis. Lymphocytes are involved in anti-tumor activity and inhibit tumor cell proliferation and migration. ${ }^{24}$ Lymphopenia can be a marker for depressed cell-mediated immunity. ${ }^{25}$ Therefore, it is known that NLR could reflect the balance between the anticancer immune system and procancer inflammatory reaction. ${ }^{26}$

There are two distinct manners to consider the trajectory changes of preoperative and postoperative NLRs. One way was to calculate the NLR-delta, which was defined as "postoperative NLR minus preoperative NLR". It was reported that a postoperative increase in NLR (positive NLR-delta value) was associated with worse survival outcomes in most of the previous studies. With respect to CRC, however, there have been contradictory results regarding the association between NLR-delta and prognosis. Guo et al showed that high NLR-delta was associated with worse OS (NLRdelta $\leq 0.037$ vs $>0.037$, HR $0.315,95 \%$ CI $0.107-0.930$, $p=0.037) .{ }^{17}$ In contrast, Li et al reported that increased NLR-delta was associated with better OS (NLR-delta $\geq 0$ vs $<0$, HR $0.223,95 \%$ CI $0.093-0.534, p=0.001){ }^{18}$

The other way was to combine the two subgroups (NLR-trend) after dividing patients into low-and highrisk groups, according to the specific values of pre- and postoperative NLRs, respectively. Although recent studies have shown that the NLR-trend showed statistical significance in predicting survival in patients with CRC, there was some discordance in survival differences among the subgroups. Chan and colleagues reported that the elevated group (low preoperative NLR changed to high postoperative NLR) showed a significantly higher risk of death (HR 
Table 2 Univariate and Multivariate Analysis of Factors Associated with Overall Survival $(n=567)$

\begin{tabular}{|c|c|c|c|c|c|}
\hline & & \multicolumn{2}{|c|}{ Univariate Analysis } & \multicolumn{2}{|c|}{ Multivariate Analysis } \\
\hline & & HR (95\% Cl) & $p$ & HR (95\% Cl) & $p$ \\
\hline Sex & $\begin{array}{l}\text { Female } \\
\text { Male }\end{array}$ & $\begin{array}{c}\text { Ref. } \\
\text { I.59 (I.15-2.20) }\end{array}$ & 0.004 & $\begin{array}{c}\text { Ref. } \\
\text { I.37 (0.98-I.92) }\end{array}$ & 0.06 \\
\hline Age (years) & $\begin{array}{l}<65 \\
\geq 65\end{array}$ & $\begin{array}{c}\text { Ref. } \\
1.66(1.23-2.23)\end{array}$ & 0.0008 & $\begin{array}{c}\text { Ref. } \\
1.29(0.94-1.77)\end{array}$ & 0.107 \\
\hline BMI $\left(\mathrm{kg} / \mathrm{m}^{2}\right)$ & $\begin{array}{l}<25 \\
\geq 25 \\
\text { No data }\end{array}$ & $\begin{array}{c}\text { Ref. } \\
0.70(0.49-1.00) \\
0.45(0.06-3.25)\end{array}$ & $\begin{array}{l}0.053 \\
0.431\end{array}$ & $\begin{array}{l}\text { NA } \\
\text { NA } \\
\text { NA }\end{array}$ & \\
\hline CEA $(\mathrm{ng} / \mathrm{mL})$ & $\begin{array}{l}<5 \\
\geq 5 \\
\text { No data }\end{array}$ & $\begin{array}{c}\text { Ref. } \\
1.86(1.37-2.52) \\
0.90(0.36-2.21)\end{array}$ & $\begin{array}{c}<0.001 \\
0.81\end{array}$ & $\begin{array}{c}\text { Ref. } \\
1.33(0.96-1.85) \\
0.67(0.26-1.68)\end{array}$ & $\begin{array}{l}0.08 \\
0.39\end{array}$ \\
\hline Tumor site & $\begin{array}{l}\text { Colon } \\
\text { Rectum }\end{array}$ & $\begin{array}{c}\text { Ref. } \\
0.8 \mathrm{I}(0.57-1.15)\end{array}$ & 0.259 & & \\
\hline Histologic grade & $\begin{array}{l}\text { GI \& G2 } \\
\text { G3 } \\
\text { Mucinous \& SRC }\end{array}$ & $\begin{array}{c}\text { Ref. } \\
1.60(0.78-3.26) \\
1.38(0.75-2.54)\end{array}$ & $\begin{array}{l}0.192 \\
0.299\end{array}$ & & \\
\hline LVI & $\begin{array}{l}\text { Absent } \\
\text { Present } \\
\text { No data }\end{array}$ & $\begin{array}{c}\text { Ref. } \\
2.09(1.52-2.87) \\
2.29(1.37-3.82)\end{array}$ & $\begin{array}{l}<0.001 \\
0.001\end{array}$ & $\begin{array}{c}\text { Ref. } \\
1.45(1.04-2.04) \\
2.00(1.16-3.44)\end{array}$ & $\begin{array}{l}0.027 \\
0.012\end{array}$ \\
\hline AJCC Stage & $\begin{array}{l}\text { I } \\
\text { III } \\
5.23(3.54-7.73)\end{array}$ & $\begin{array}{c}\& \text { II } \\
1.35(0.93-1.96) \\
<0.001\end{array}$ & $\begin{array}{c}\text { Ref. } \\
0.109 \\
4.11(2.67-6.33)\end{array}$ & $\begin{array}{c}1.90(1.23-2.94) \\
<0.001\end{array}$ & $\begin{array}{l}\text { Ref. } \\
0.003\end{array}$ \\
\hline Complications & $\begin{array}{l}\text { No } \\
\text { Yes }\end{array}$ & $\begin{array}{c}\text { Ref. } \\
1.43(1.02-1.99)\end{array}$ & 0.036 & $\begin{array}{l}\text { NA } \\
\text { NA }\end{array}$ & \\
\hline Chemotherapy & $\begin{array}{l}\text { No } \\
\text { Yes }\end{array}$ & $\begin{array}{c}\text { Ref. } \\
0.39(0.29-0.52)\end{array}$ & $<0.001$ & $\begin{array}{c}\text { Ref. } \\
0.37(0.26-0.53)\end{array}$ & $<0.001$ \\
\hline NLR-pre & $\begin{array}{l}\text { Low } \\
\text { High }\end{array}$ & $\begin{array}{c}\text { Ref. } \\
1.67(1.24-2.26)\end{array}$ & 0.0007 & $\begin{array}{l}\text { NA } \\
\text { NA }\end{array}$ & \\
\hline NLR-post & $\begin{array}{l}\text { Low } \\
\text { High }\end{array}$ & $\begin{array}{c}\text { Ref. } \\
1.56(1.16-2.10)\end{array}$ & 0.003 & $\begin{array}{l}\text { NA } \\
\text { NA }\end{array}$ & \\
\hline NLR-trend & $\begin{array}{l}\text { GI } \\
\text { G2 } \\
\text { G3 } \\
\text { G4 }\end{array}$ & $\begin{array}{c}\text { Ref. } \\
2.18(1.38-3.46) \\
2.15(1.43-3.23) \\
2.28(1.50-3.46)\end{array}$ & $\begin{array}{l}0.0008 \\
0.0002 \\
0.0001\end{array}$ & $\begin{array}{c}\text { Ref. } \\
1.86(1.17-2.97) \\
2.02(1.33-3.08) \\
2.00(1.30-3.10)\end{array}$ & $\begin{array}{l}0.0084 \\
0.0009 \\
0.0016\end{array}$ \\
\hline NLR-delta & $\begin{array}{l}\text { Low } \\
\text { High }\end{array}$ & $\begin{array}{c}\text { Ref. } \\
0.68(0.5-0.94)\end{array}$ & 0.02 & $\begin{array}{l}\text { NA } \\
\text { NA }\end{array}$ & \\
\hline
\end{tabular}

Abbreviations: BMI, body mass index; CEA, carcinoembryonic antigen; SRC, signet ring cell; LVI, lymphovascular invasion; AJCC, American Joint Committee on Cancer; $\mathrm{HR}$, hazard ratio; $\mathrm{Cl}$, confidence interval.

2.156, 95\% CI 1.453-3.199) than the persistently low NLR group using 587 stage I-IV CRC patients. ${ }^{10}$ However, another study by Yasui and colleagues reported that the persistently low NLR group and exacerbation group (preoperative low to postoperative high NLR) showed no difference in survival in patients with stage III CRC. ${ }^{9}$

To the best of our knowledge, the prognostic impact of NLR-delta and NLR-trend has been investigated in a limited manner. Cui et al analyzed both NLR-delta and 


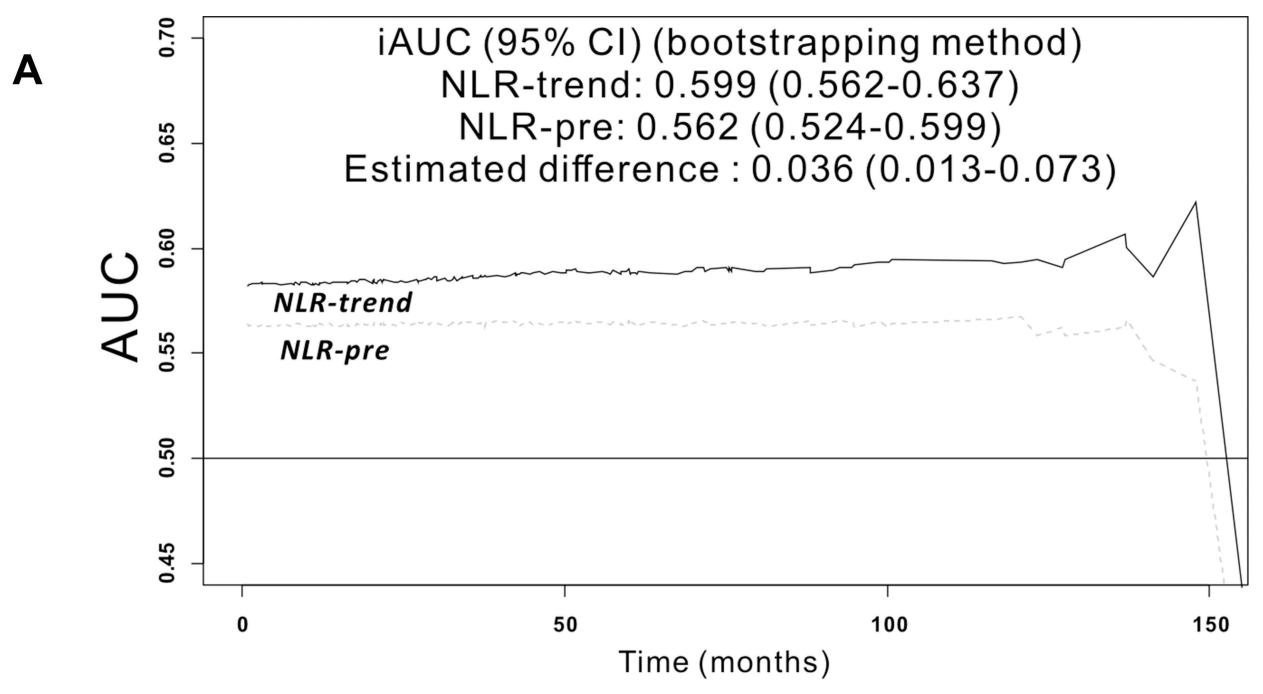

B
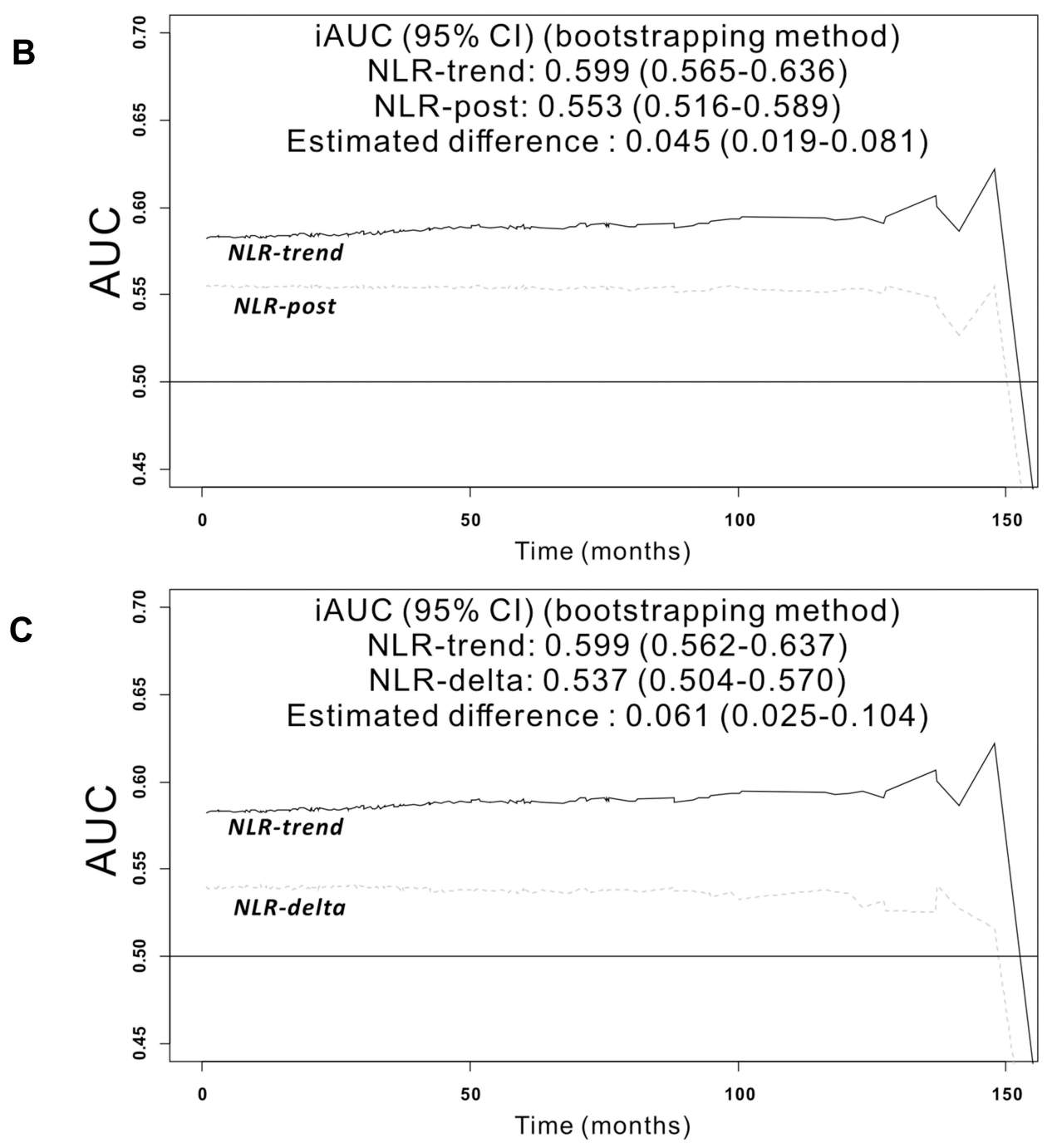

Figure 2 Predictive accuracy of the model during follow-up period was measured using the iAUC, a weighted mean of AUC over follow-up period. The time-dependent receiver operating characteristics curve of the NLR-trend was superior to that of NLR-pre (A) (bootstrap iAUC mean difference =0.036; 95\% Cl 0.013-0.073), NLR-post (B) (bootstrap iAUC mean difference $=0.045 ; 95 \% \mathrm{Cl} 0.019-0.08 \mathrm{I})(\mathbf{B})$ and NLR-delta (C) (bootstrap iAUC mean difference $=0.06 \mathrm{I}$; 95\% Cl 0.025-0.104). 
NLR groupings to determine the association with progression-free survival (PFS) and reported that NLR-delta could not predict PFS, while grouping low-low and low-high subgroups had better PFS. ${ }^{27}$ Nevertheless, the time point for postoperative NLR measurement in that study was described as at least 7 days after the surgery, which was too broad to be a standard.

In this context, the strength of our study is that the different NLR-related parameters were included simultaneously, and the discriminatory performance was statistically compared using iAUC. We set the optimal cut-off value of NLR-delta as -1.08 ; however, this dichotomy was not associated with survival in the multivariate analysis. With respect to NLR-trend, our analysis showed that G2 (low NLR-pre and high NLR-post) showed a significantly higher risk of death (HR 1.86, 95\% CI 1.17-2.97, $p=0.0084$ ) than G1, in concordance with the results of Chan et al. These results indicate that the elevated NLR group in both perioperative periods showed poor survival outcomes compared with the persistently low NLR group. This agrees with our previous analysis in patients with locally advanced rectal cancer, which demonstrated that a persistently low NLR during preoperative chemoradiotherapy treatment was associated with better disease-free survival than the other groups (HR 0.37 , $95 \%$ CI $0.15-0.89, p=0.033) .{ }^{28}$ In addition, we found that the NLR-trend showed better discriminatory performance than the other NLR-related parameters such as NLR-pre, NLRpost and NLR-delta.

Another value of our study is that the timing of postoperative NLR measurement was clearly defined. Previous studies did not mention the timing of postoperative NLR tests, nor did they examine the immediate postoperative periods, 1-4 months or 3-6 months after surgery (Supplementary Tables $2 \& 3$ ). In our study, we strictly selected patients whose postoperative NLR test was performed between 21-56 days postoperatively, before starting postoperative chemotherapy. This time point is thought to be an appropriate time to measure the patient's intrinsic NLR level, in which the effect of surgery-induced NLR rise disappears and the effect of the start of chemotherapy is not involved. ${ }^{10} \mathrm{~A}$ meta-analysis of 14 studies showed that a delay of $>8$ weeks in starting adjuvant chemotherapy is associated with a higher relative risk of death (HR 1.20, 95\% CI $1.15-1.26, p=0.001) .{ }^{29}$ Thus, starting chemotherapy within 8 weeks after surgery is highly recommended in the guidelines. ${ }^{20,29}$ If it is possible to make a decision on the implementation of chemotherapy by comparing the
NLR measured pre- and postoperatively, it would provide great help for the patients. In our study, the group with consistently low NLR before and after surgery showed the best prognosis. In view of results of our study, in order to use NLR as a clinical indicator, both preoperative and postoperative NLR should be considered together. The inflammatory reaction after the surgery derived from postoperative complications or residual tumor burden can effects the prognosis even in patients with low preoperative NLR. This observation may be clinically useful in decision making regarding whether a patient with CRC who underwent surgical resection can omit postoperative chemotherapy, although further investigation is required to confirm our hypothesis.

Our study has several limitations. Firstly, it was a retrospective single-center study, meaning selection bias is inevitable. There is no consensus on the cut-off values of NLR-related parameters, and therefore the optimized values for our cohort were selected. This might hinder the generalized application of our results. However, if we wish to apply a predefined NLR threshold, the clinical significance of NLR should be the same, which would be quite difficult to assume considering different patient or disease characteristics. ${ }^{30}$ During postoperative periods, NLR can be elevated due to various causes. One cause of this could be systemic inflammation due to postoperative complications. In our study, as mentioned earlier, to minimize the effect of surgery itself on the postoperative NLR value, the time point of postoperative laboratory sampling was highly specified. In this context, to prevent bias caused by neutrophil elevation due to postoperative complications, our study excluded patients whose discharge date was later than the date that the postoperative NLR results were collected. In some aspects, these excluded patients may have a worse prognosis, which could cause another selection bias.

In conclusion, our study revealed that combining preoperative and postoperative NLR acts synergistically to improve prognostic accuracy compared with preoperative, postoperative alone, or NLR-delta, and patients with persistently low NLR values through perioperative periods showed the best prognosis. Considering the recommended period of post-operative chemotherapy, the clinical implication of stratification as a suitable indicator for adding adjuvant chemotherapy and a guide to patient-specific therapeutic interventions need to be investigated. 


\section{Acknowledgment}

We would like to thank Editage for English language editing.

\section{Disclosure}

The authors report no conflicts of interest in this work.

\section{References}

1. Siegel RL, Miller KD, Jemal A. Cancer statistics, 2020. CA Cancer J Clin. 2020;70(1):7-30. doi:10.3322/caac.21590

2. Hong S, Won YJ, Park YR, Jung KW, Kong HJ, Lee ES. Cancer statistics in Korea: incidence, mortality, survival, and prevalence in 2017. Cancer Res Treat. 2020;52(2):335-350. doi:10.4143/ crt.2020.206

3. Faratian D, Clyde RG, Crawford JW, Harrison DJ. Systems pathology-taking molecular pathology into a new dimension. Nat Rev Clin Oncol. 2009;6(8):455-464. doi:10.1038/nrclinonc.2009.102

4. Sagaert X, Vanstapel A, Verbeek S. Tumor heterogeneity in colorectal cancer: what do we know so far? Pathobiology. 2018;85(12):72-84. doi:10.1159/000486721

5. Tuomisto AE, Mäkinen MJ, Väyrynen JP. Systemic inflammation in colorectal cancer: underlying factors, effects, and prognostic significance. World $J$ Gastroenterol. 2019;25(31):4383-4404. doi:10.3748/wjg.v25.i31.4383

6. Hu B, Yang XR, Xu Y, et al. Systemic immune-inflammation index predicts prognosis of patients after curative resection for hepatocellular carcinoma. Clin Cancer Res. 2014;20(23):6212-6222. doi:10.1158/1078-0432.Ccr-14-0442

7. Templeton AJ, McNamara MG, Šeruga B, et al. Prognostic role of neutrophil-to-lymphocyte ratio in solid tumors: a systematic review and meta-analysis. $J$ Natl Cancer Inst. 2014;106(6):dju124. doi:10.1093/jnci/dju124

8. Cupp MA, Cariolou M, Tzoulaki I, Aune D, Evangelou E, BerlangaTaylor AJ. Neutrophil to lymphocyte ratio and cancer prognosis: an umbrella review of systematic reviews and meta-analyses of observational studies. BMC Med. 2020;18(1):360. doi:10.1186/s12916-020-01817-1

9. Yasui K, Shida D, Nakamura Y, Ahiko Y, Tsukamoto S, Kanemitsu Y. Postoperative, but not preoperative, inflammation-based prognostic markers are prognostic factors in stage III colorectal cancer patients. Br J Cancer. 2020;124(5):933-941. doi:10.1038/s41416-020-01189-6

10. Chan JCY, Diakos CI, Chan DLH, et al. A longitudinal investigation of inflammatory markers in colorectal cancer patients perioperatively demonstrates benefit in serial remeasurement. Ann Surg. 2018;267 (6):1119-1125. doi:10.1097/sla.0000000000002251

11. Micaela R, Lucas C, Franco C, Federico C, Agustín D, David S. Dynamic perioperative variation of neutrophil-to-lymphocyte ratio as an independent prognosis factor following lobectomy for NSCLC. Updates Surg. 2021;73(4):1567-1574. doi:10.1007/s13304-020-00936-x

12. Guo M, Li W, Li B, et al. Prognostic value of delta inflammatory biomarker-based nomograms in patients with inoperable locally advanced NSCLC. Int Immunopharmacol. 2019;72:395-401. doi:10.1016/j.intimp.2019.04.032

13. Peng W, Li C, Wen TF, et al. Neutrophil to lymphocyte ratio changes predict small hepatocellular carcinoma survival. J Surg Res. 2014;192(2):402-408. doi:10.1016/j.jss.2014.05.078

14. Seong YW, Han SJ, Jung W, et al. Perioperative change in neutrophil-to-lymphocyte ratio (NLR) is a prognostic factor in patients with completely resected primary pulmonary sarcomatoid carcinoma. J Thorac Dis. 2019;11(3):819-826. doi:10.21037/jtd.2019.02.02
15. Yamazaki H, Sugino K, Matsuzu K, et al. Inflammatory biomarkers and dynamics of neutrophil-to-lymphocyte ratio in anaplastic thyroid carcinoma. Endocrine. 2020;70(1):115-122. doi:10.1007/s12020-02002313-5

16. Min KW, Kwon MJ, Kim DH, et al. Persistent elevation of postoperative neutrophil-to-lymphocyte ratio: a better predictor of survival in gastric cancer than elevated preoperative neutrophil-to-lymphocyte ratio. Sci Rep. 2017;7(1):13967. doi:10.1038/s41598-017-13969-x

17. Guo D, Han A, Jing W, et al. Preoperative to postoperative change in neutrophil-to-lymphocyte ratio predict survival in colorectal cancer patients. Future Oncol. 2018;14(12):1187-1196. doi:10.2217/fon2017-0659

18. Li Z, Zhao R, Cui Y, Zhou Y, Wu X. The dynamic change of neutrophil to lymphocyte ratio can predict clinical outcome in stage I-III colon cancer. Sci Rep. 2018;8(1):9453. doi:10.1038/s41598-01827896-y

19. Bayraktar S, Bayraktar UD, Rocha-Lima CM. Timing of adjuvant and neoadjuvant therapy in colorectal cancers. Clin Colorectal Cancer. 2010;9(3):144-149. doi:10.3816/CCC.2010.n.019

20. Van Cutsem E, Cervantes A, Adam R, et al. ESMO consensus guidelines for the management of patients with metastatic colorectal cancer. Ann Oncol. 2016;27(8):1386-1422.

21. Camp RL, Dolled-Filhart M, Rimm DL. X-tile: a new bio-informatics tool for biomarker assessment and outcome-based cut-point optimization. Clin Cancer Res. 2004;10(21):7252-7259. doi:10.1158/1078-0432.Ccr-04-0713

22. Song Y, Yang Y, Gao P, et al. The preoperative neutrophil to lymphocyte ratio is a superior indicator of prognosis compared with other inflammatory biomarkers in resectable colorectal cancer. $B M C$ Cancer. 2017;17(1):744. doi:10.1186/s12885-017-3752-0

23. Wu L, Saxena S, Singh RK. Neutrophils in the tumor microenvironment. Adv Exp Med Biol. 2020;1224:1-20. doi:10.1007/978-3-030-35723-8_1

24. Jakubowska K, Koda M, Kisielewski W, Kańczuga-Koda L, Grudzińska M, Famulski W. Pre- and postoperative neutrophil and lymphocyte count and neutrophil-to-lymphocyte ratio in patients with colorectal cancer. Mol Clin Oncol. 2020;13(5):56. doi:10.3892/mco.2020.2126

25. Zahorec R. Ratio of neutrophil to lymphocyte counts-rapid and simple parameter of systemic inflammation and stress in critically ill. Bratisl Lek Listy. 2001;102(1):5-14.

26. Li MX, Liu XM, Zhang XF, et al. Prognostic role of neutrophil-tolymphocyte ratio in colorectal cancer: a systematic review and meta-analysis. Int J Cancer. 2014;134(10):2403-2413. doi:10.1002/ ijc. 28536

27. Cui M, Xu R, Yan B. A persistent high neutrophil-to-lymphocyte ratio predicts poor prognosis in patients with colorectal cancer undergoing resection. Mol Clin Oncol. 2020;13(5):63. doi:10.3892/ mco.2020.2133

28. Cha YJ, Park EJ, Baik SH, Lee KY, Kang J. Prognostic impact of persistent lower neutrophil-to-lymphocyte ratio during preoperative chemoradiotherapy in locally advanced rectal cancer patients: a propensity score matching analysis. PLoS One. 2019;14(3): e0214415. doi:10.1371/journal.pone.0214415

29. Des Guetz G, Nicolas P, Perret GY, Morere JF, Uzzan B. Does delaying adjuvant chemotherapy after curative surgery for colorectal cancer impair survival? A meta-analysis. Eur J Cancer. 2010;46 (6):1049-1055. doi:10.1016/j.ejca.2010.01.020

30. Howard R, Kanetsky PA, Egan KM. Exploring the prognostic value of the neutrophil-to-lymphocyte ratio in cancer. Sci Rep. 2019;9 (1):19673. doi:10.1038/s41598-019-56218-z 


\section{Publish your work in this journal}

The Journal of Inflammation Research is an international, peerreviewed open-access journal that welcomes laboratory and clinical findings on the molecular basis, cell biology and pharmacology of inflammation including original research, reviews, symposium reports, hypothesis formation and commentaries on: acute/chronic inflammation; mediators of inflammation; cellular processes; molecular mechanisms; pharmacology and novel anti-inflammatory drugs; clinical conditions involving inflammation. The manuscript management system is completely online and includes a very quick and fair peerreview system. Visit http://www.dovepress.com/testimonials.php to read real quotes from published authors. 